\title{
The BGS magnetic field candidate models for the 12th generation IGRF
}

\author{
Brian Hamilton*, Victoria A Ridley, Ciarán D Beggan and Susan Macmillan
}

\begin{abstract}
We describe the candidate models submitted by the British Geological Survey for the 12th generation International Geomagnetic Reference Field. These models are extracted from a spherical harmonic 'parent model' derived from vector and scalar magnetic field data from satellite and observatory sources. These data cover the period 2009.0 to 2014.7 and include measurements from the recently launched European Space Agency (ESA) Swarm satellite constellation. The parent model's internal field time dependence for degrees 1 to 13 is represented by order 6 B-splines with knots at yearly intervals. The parent model's degree 1 external field time dependence is described by periodic functions for the annual and semi-annual signals and by dependence on the 20-min Vector Magnetic Disturbance index. Signals induced by these external fields are also parameterized. Satellite data are weighted by spatial density and by two different noise estimators: (a) by standard deviation along segments of the satellite track and (b) a larger-scale noise estimator defined in terms of a measure of vector activity at the geographically closest magnetic observatories to the sample point. Forecasting of the magnetic field secular variation beyond the span of data is by advection of the main field using core surface flows.
\end{abstract}

Keywords: IGRF; Geomagnetic field; Geomagnetic secular variation; Swarm

\section{Background}

The International Geomagnetic Reference Field (IGRF) is a widely used model of the Earth's main magnetic field that is updated every 5 years. Under the auspices of the International Association of Geomagnetism and Aeronomy (IAGA), an open invitation is made for the submission of candidate models. These models are then evaluated, and from them, the next revision of the IGRF is constructed. IGRF-12, the 12th generation model, includes three new sets of coefficients: two main field (MF) models to degree and order 13 at epochs 2010.0 and 2015.0 and an average secular variation (SV) model valid from 2015.0 to 2020.0 to degree and order 8. The production of our British Geological Survey (BGS) candidate models was carried out in three steps: (a) selection of data from Ørsted and CHAMP, the new European Space Agency (ESA) Swarm mission launched in November 2013, and ground magnetic observatories; (b) fitting and evaluation of the parent model; and (c) derivation of the IGRF-12 candidate MF and SV coefficients. Since the production

*Correspondence: bham@bgs.ac.uk

British Geological Survey, West Mains Road, Edinburgh EH9 3LA, UK of the BGS IGRF-11 candidate models (Hamilton et al. 2010), the most significant changes are the parameterization of the secular variation using order $6 \mathrm{~B}$-splines, the use of core flow advection to predict the secular variation, and the use of satellite data from the ESA Swarm mission.

Since the launch of Ørsted in 1999 and CHAMP the following year, a large number of vector and scalar measurements with excellent geographic coverage have been collected. Continuous satellite vector data ceased temporarily with the end of the CHAMP mission in September 2010, though vector data from ground-based observatories have continued uninterrupted, providing a high-quality data set throughout. In November 2013, ESA launched its three-satellite Swarm constellation mission. This provided an unprecedented density of global vector data, which have greatly benefited our IGRF-12 candidate models. Together these data provide a large data set for modelling.

However, in addition to the core and large-scale lithospheric sources that are the focus of the IGRF-12 modelling effort, this large compilation of satellite and

\section{Springer}

(c) 2015 Hamilton et al; licensee Springer. This is an Open Access article distributed under the terms of the Creative Commons Attribution License (http://creativecommons.org/licenses/by/4.0), which permits unrestricted use, distribution, and reproduction in any medium, provided the original work is properly credited. 
observatory data contains fields produced from other sources. In particular, the ionospheric current system, ring current, partial ring current, and induced currents in the Earth can be difficult to co-estimate and separate from the desired core field signal. To avoid contamination, measurements significantly affected by these sources must be removed from the data set through careful selection prior to modelling.

Even with rigorous selection, contamination from unwanted sources remains a problem, particularly at high latitudes. For this reason, scalar data have often been used at high latitudes in preference to vector components which tend to be more sensitive to high-latitude current systems. We use only observatory pseudo-scalar data (vector data projected onto the field from an a priori main field model based on a similar parameterisation to that described in the 'Model parameterization and estimation' section) at higher latitudes. This also has the effect of making the co-estimation of the observatory biases easier by maintaining the linear relationship between data and model coefficients. However, for the satellite data, we use vector components in preference to scalar data at all latitudes wherever they are available. At the same time, we quantify the increase in noise in the data when we fit our parent model. This is achieved by using a combination of two noise estimators: an along-track standard deviation calculated for each component from short segments of the satellite data and a larger-scale estimator of the vector disturbance using observatories closest to the data sample (Thomson et al. 2010).

Within the 'Methods' section, we describe the data used and their selection criteria and briefly outline the data weighting scheme. We also describe the parent model and the process used to fit its parameters, and we describe the core-flow method of modelling and predicting secular variation. In the 'Results and discussion' section, we evaluate the model coefficients and derive the DGRF, IGRF, and secular variation candidate models and compare our model with the other IGRF-12 candidate models. We provide concluding remarks in the 'Conclusions' section.

\section{Methods}

\section{Data selection and weighting}

We use CHAMP (calibration level version 51) scalar and vector data between 2009.0 and 2010.7. Ørsted scalar data are used between 2009.0 and 2013.5 (only scalar data are available since 2005.9) and data from the three Swarm satellites (Alpha, Bravo, and Charlie, versions 0301 and 0302) from 2013.9 to 2014.7. To reduce the quantity of data to manageable levels, the satellite data were subsampled at every 20th datum giving a sample frequency of approximately once every $20 \mathrm{~s}$. In order to reduce contamination from magnetospheric and ionospheric sources, data were selected according to the criteria in Table 1.

In addition to these criteria, manual checks were made for the Swarm Level 1b data using the Quick Look products (Beggan et al. 2013). These revealed spurious signals and spikes in some day files (see Table 1) which were removed from the selected data. The distribution of the selected satellite data with time is shown in Figure 1.

Observatory hourly mean data were taken from 148 observatories between 2009.0 and 2014.7 held at the World Data Centre for Geomagnetism. Known jumps, identified by the operators of the observatories, were corrected for and some poor-quality data were eliminated manually. These cleaned data (without any further selection) are regularly updated and made available by BGS to ESA as the Swarm 'AUX_OBS' product (Macmillan and Olsen 2013) for use by the wider community. The data were then selected according to the criteria in Table 1.

In a change from our IGRF-11 candidates (Hamilton et al. 2010), we do not select data according to zenith angle. Mid- and low-latitude day-side ionospheric signals are still avoided using local time selection, but high-latitude day-lit data are permitted. This avoids large seasonal gaps in polar data, which tests have shown to produce unwanted polar signals in the model. Such data gaps are undesirable given the relatively few data available in the 2011 to 2014 period (see Figure 1). In future models, with increased data coverage from Swarm, and improved damping in the model estimation, these data may again be rejected (as they are in some other model, e.g. CHAOS-4 (Olsen et al. 2014)).

After selection, those observatories contributing to the model are shown in Figure 2. For high geomagnetic dipole latitudes $\left(>50^{\circ}\right.$ and $\left.<-50^{\circ}\right)$, observatory vector data were projected onto an a priori model vector, and the resulting pseudo-scalar data were used in the inversion. For other latitudes, vector data were used. From Figure 1, it can be seen that the observatory data provide a continuous time series compared to the satellite data. This is desirable for the robust time parameterization of the model.

The final data set consists of 281,967 Ørsted, 1,353,331 CHAMP, 455,840 Swarm A, 428,868 Swarm B, 408,362 Swarm C, and 354,362 observatory data giving a total of $3,435,253$ individual data points (now counting each vector component separately). CHAMP vector and Ørsted scalar data dominate early in the time series, providing good global coverage. After the end of the CHAMP mission in late 2010, satellite data become scarcer, and vector satellite data are completely absent until the launch of Swarm in late 2013. However, Ørsted does continue to provide global coverage of scalar data, albeit with gaps 


\section{Table 1 Data selection criteria}

\begin{tabular}{|c|c|c|}
\hline Quantity/index & Satellite & Observatory \\
\hline \multirow[t]{2}{*}{$\overline{K p}$} & $\leq 2-$ now & $\leq 2+$ \\
\hline & $\leq 2-$ previous $3 \mathrm{~h}$ & \\
\hline$|d D s t / d t|$ & $\leq 5 \mathrm{nT} / \mathrm{h}$ & $\leq 5 \mathrm{nT} / \mathrm{h}$ \\
\hline IMF $B_{x}(n T)$ & $\geq-10$ and $\leq+10$ & - \\
\hline IMF B $B_{y}(n T)$ & $\geq-3$ and $\leq+3$ & - \\
\hline $\operatorname{MFF} B_{Z}(n T)$ & $\geq 0$ and $\leq+6$ & $\geq-2$ \\
\hline Solar wind speed (km/s) & $\leq 450$ & - \\
\hline \multirow[t]{2}{*}{ Local time (hh:mm) } & $\geq 22: 30$ and $\leq 05: 00$ & $\geq 01: 00$ and $\leq 02: 00$ \\
\hline & for geomagnetic latitudes between $\pm 50^{\circ}$ & \\
\hline |Observed field - IGRF-11| (nT) & $\leq 700$ & - \\
\hline |Scalar F-F from vector| (nT) & $\leq 2$ & - \\
\hline Manual rejection & Yes, see below & Yes \\
\hline
\end{tabular}

Rejected Swarm Level 1b data:

$$
\begin{aligned}
& \text { SW_OPER_MAGA_LR_1B_20140104T000000_20140104T235959_0302_MDR_MAG_LR } \\
& \text { SW_OPER_MAGA_LR_1B_20140129T000000_20140129T235959_0302_MDR_MAG_LR } \\
& \text { SW_OPER_MAGA_LR_1B_20140130T000000_20140130T235959_0302_MDR_MAG_LR } \\
& \text { SW_OPER_MAGA_LR_1B_20140206T000000_20140206T235959_0302_MDR_MAG_LR } \\
& \text { SW_OPER_MAGB_LR_1B_20140101T000000_20140101T235959_0302_MDR_MAG_LR } \\
& \text { SW_OPER_MAGC_LR_1B_20131218T000000_20131218T235959_0302_MDR_MAG_LR } \\
& \text { SW_OPER_MAGC_LR_1B_20140325T000000_20140325T235959_0302_MDR_MAG_LR } \\
& \text { SW_OPER_MAGC_LR_1B_20140326T000000_20140326T235959_0302_MDR_MAG_LR } \\
& \text { SW_OPER_MAGC_LR_1B_20140404T000000_20140404T235959_0302_MDR_MAG_LR } \\
& \text { SW_OPER_MAGC_LR_1B_20140408T000000_20140408T235959_0302_MDR_MAG_LR } \\
& \text { SW_OPER_MAGC_LR_1B_20140409T000000_20140409T235959_0302_MDR_MAG_LR } \\
& \text { SW_OPER_MAGC_LR_1B_20140318T000000_20140318T235959_0301_MDR_MAG_LR } \\
& \text { SW_OPER_MAGC_LR_1B_20140319T000000_20140319T235959_0301_MDR_MAG_LR } \\
& \text { SW_OPER_MAGC_LR_1B_20140401T000000_20140401T235959_0301_MDR_MAG_LR }
\end{aligned}
$$

All criteria must be passed for a datum to be selected. Manual rejection refers to rejection of data based on visual inspection of the time series. The manually rejected Level $1 \mathrm{~b}$ Swarm data can be uniquely identified by the file names given.

in temporal coverage. Throughout the period, observatories provided continuous vector and scalar data. Since the launch of Swarm, global coverage of continuous satellite vector measurements has returned.

Given the variability in data coverage since 2009, we are most confident in model snapshots that are dependent on data early and late in the time series. Fortunately, the DGRF and IGRF models are at 2010.0 and 2015.0, respectively. However, our estimates of the secular variation from modelling the core flow (see the 'Derivation of IGRF candidate models' section) benefit from knowledge of the field evolution throughout this 5-year period. These core flow models are used not only to estimate the secular variation from 2015 to 2020 but also to estimate the IGRF coefficients at 2015.0. For this reason, the Ørsted and observatory data through 2011 to 2014 hold valuable information, especially in the absence of globally available vector data.

The selected satellite data were individually weighted using two 'noise' estimators which we briefly outline here (for a detailed description, see Thomson et al. (2010)). Firstly, we use a measure of local magnetic activity derived from the standard deviation (SD) along short segments (20 samples, approximately $150 \mathrm{~km}$ ) of satellite track. Secondly, we employ a larger-scale noise estimator (the 'LAVA' index) derived from the activity measured at the geographically closest magnetic observatories to the sample point. This results in the down-weighting of the vector data at high latitudes, particularly in the auroral zones. We find the technique produces a core and lithospheric field model, MEME08 (Thomson et al. 2010), that compares well with other similar models of the period up to 


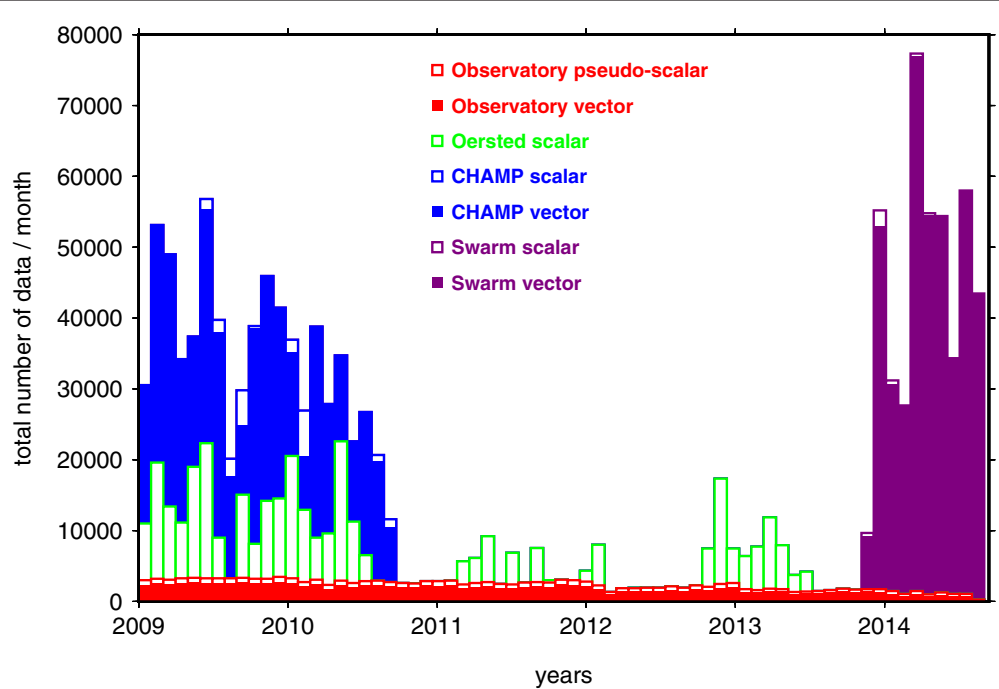

Figure 1 Temporal distribution of selected data from each source used to fit the parent model. In these plots, a triplet of vector components or a scalar measurement are both counted as a single measurement.

about degree and order 60 . We also apply weighting based on instrument accuracy and zenith angle (to account for ionospheric field contamination) up to a maximum of 6 $\mathrm{nT}$ and also $1^{\circ}$ tesseral weighting to account for different spatial data densities, in the same manner as Lesur et al. (2005).

For the observatory data weighting, we use a simpler scheme based on a scaling of the instrument accuracy and zenith angle weighting up to a maximum of $10 \mathrm{nT}$. Unlike the satellite weighting, this scheme is a priori and not based on samples of data from each observatory. Although we select from a wide global distribution of observatories, these data still represent a spatially inhomogeneous distribution. This could bias the model fit towards their locations depending on the relative sensitivity of the model to the different data sources, which is a separate effect from the noise recorded in the data. Any attempt to mitigate this potential issue must acknowledge that the observatory data will inevitably dominate where the satellite data are scarce.

Conceding to these complications, we simply apply a single scaling to all the observatory data variances to achieve an overall relative weighting of satellite to observatory data of approximately 10:1 chosen from an analysis of test models with different ratios (not shown). The 'weight' of each source is estimated from the sum of the inverse of the diagonal (variance) elements of the data covariance matrix and is applied to the data from each source that passes the selection criteria defined above. Note that Ørsted's covariance matrix contains offdiagonal entries to account for the anisotropic error introduced due to the presence of only one star camera (see Lesur et al. (2005)). The weight estimates for observatory and satellite data versus time are shown in Figure 3, which varies considerably for satellite data due primarily to the large variations in the amount of selected data. This

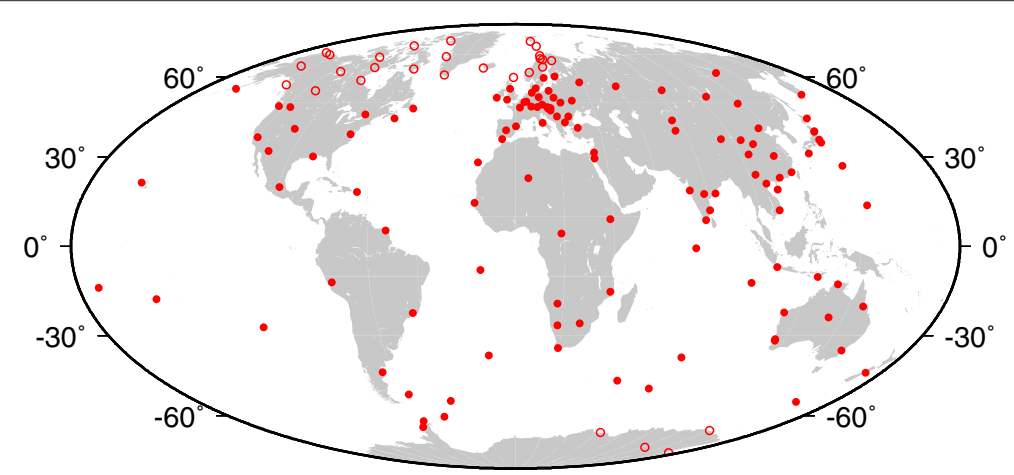

Figure 2 Spatial distribution of selected observatories contributing data used to fit the parent model. Solid and hollow markers indicate sources of vector and pseudo-scalar data, respectively. 
treatment is currently based on an ad hoc argument and can be improved by accounting for the time dependence of the data densities as well as a more direct estimate of the noise at each observatory (e.g. Finlay et al. (2012)).

In addition to the satellite and observatory data selected for direct use in the modelling, minute mean definitive and quasi-definitive observatory data are used to estimate the Vector Magnetic Disturbance index (Thomson and Lesur 2007). These indices are then used to co-estimate the largest-scale rapidly varying magnetospheric signals still present in the satellite and observatory data after the selection described in Table 1.

\section{Model parameterization and estimation}

The model parameterization follows Hamilton et al. (2010), with some changes, and consists of approximately 6,000 separate parameters split across four main terms:

$$
V=V^{\text {int }}+V^{\text {ext }}+V^{\mathrm{vmd}}+V^{\text {ann }}
$$

where $V^{\text {int }}$ describes the time-varying core and static lithospheric field:

$$
\begin{aligned}
V^{\mathrm{int}}(\theta, \phi, r, t)= & a \sum_{l=1}^{13} \sum_{m=-l}^{+l} g_{l}^{m}(t)\left(\frac{a}{r}\right)^{l+1} Y_{l}^{m}(\theta, \phi) \\
& +a \sum_{l=14}^{55} \sum_{m=-l}^{+l} g_{l}^{m}\left(\frac{a}{r}\right)^{l+1} Y_{l}^{m}(\theta, \phi)
\end{aligned}
$$

$r$ is the radius, $a$ is the reference radius taken to be the mean Earth radius $(6,371.2 \mathrm{~km})$, and $\phi$ and $\theta$ are geocentric, Earth-fixed, longitude and co-latitude, respectively. $g_{l}^{m}$ are the Gauss coefficients associated with the Schmidt semi-normalised spherical harmonic functions $Y_{l}^{m}(\theta, \phi)$ of degree $l$ and order $m$ using the convention of negative $m$ for sine longitude terms and non-negative for cosine terms. In a change from Hamilton et al. (2010), we now describe the time dependence of $g_{l}^{m}(t)$ by order 6 B-splines, which is consistent with our regularisation scheme (following Lesur et al. (2010)) using annual knots from 2008.7 to 2014.7 with repeated end knots.

$V^{\text {ext }}$ is the scalar potential associated with the external terms that consists of degree 1 coefficients, $q_{1}^{m}(t)$, with piecewise linear time dependence over the same periods defined by the knots of $V^{\text {int }}$. Their parameters should account for any long period, largest-scale external fields not represented by the VMD-dependent terms (see Equation 4):

$$
V^{\mathrm{ext}}(\theta, \phi, r, t)=a \sum_{m=-1}^{+1} q_{1}^{m}(t)\left(\frac{r}{a}\right) Y_{1}^{m}(\theta, \phi)
$$

$V^{\text {vmd }}$ consists of terms that are dependent on the VMD index, an index of magnetospheric field activity that we believe is better able to parameterize the largestscale magnetospheric field than the Dst index. VMD is described in detail in Thomson and Lesur (2007):

$$
\begin{aligned}
V^{\mathrm{vmd}}(\theta, \phi, r, t, \mathrm{VMD})= & a \sum_{m=-1}^{+1}\left\{b_{1}^{m}(t) \mathrm{VMD}_{\mathrm{ext}}^{m}(t)\left(\frac{r}{a}\right)\right. \\
& \left.+c_{1}^{m}(t) \mathrm{VMD}_{\text {int }}^{m}(t)\left(\frac{a}{r}\right)^{2}\right\} Y_{1}^{m}(\theta, \phi)
\end{aligned}
$$

$\mathrm{VMD}_{\text {ext }}^{m}$ and $\mathrm{VMD}_{\text {int }}^{m}$ are the external and internal VMD indices that are interpolated at the time of each satellite and observatory datum from a degree 1 spherical harmonic model. This model is fitted in an Earth-fixed coordinate system with time dependence given by cubic B-splines with knots every hour, produced from observatory data. The IGRF parent model estimates separate sets of coefficients, $b_{1}^{m}(t)$ and $c_{1}^{m}(t)$, every 3 months, the same period over which VMD indices are fit to detrended observatory data.

Finally, $V^{\text {ann }}$ consists of terms that model the annual, semi-annual, and diurnal variations:

$$
\begin{aligned}
V^{\mathrm{ann}}(\theta, \phi, \tilde{\phi}, r, t)= & a \sum_{m=-1}^{+1}\left[e_{1}^{m}(t)\left(\frac{r}{a}\right)+i_{1}^{m}(t)\left(\frac{a}{r}\right)^{2}\right] Y_{1}^{m}(\theta, \phi) \\
& +a \sum_{m=-1}^{+1} \epsilon_{1}^{m}(t)\left(\frac{r}{a}\right) Y_{1}^{m}(\theta, \tilde{\phi})
\end{aligned}
$$

where $e_{1}^{m}$ and $i_{1}^{m}$ are, respectively, the external and internal coefficients consisting of cosine and sine annual and semiannual terms. $\epsilon_{1}^{m}$ is the external 24-h term, which is expressed in terms of sun-synchronous longitude, $\tilde{\phi}$, rather than Earth-fixed geographic longitude and consists of a constant and cosine and sine annual terms.

Our parameterization of all the external and induced fields is to degree 1 only. In contrast, other recent global magnetic field models (e.g. Lesur et al. (2010)); Maus et al. (2010), and Olsen et al. (2014)) parameterize the external field up to degree 2, either through co-estimation or a priori models. The Comprehensive Model family of models (e.g. Sabaka et al. (2002)) allow for co-estimation to even higher degrees. The presence of higher-degree magnetospheric sources is expected from the knowledge of those current systems and have been estimated by (Lühr and Maus 2010) up to degree 2 for relatively quiet geomagnetic conditions. However, we do account for the dominant/largest-scale magnetospheric signals and note that the dominant unmodelled sources, as seen in the residuals, are in the auroral regions and would still not be adequately co-estimated by a parameterization up to degree 2 (discussed below). 


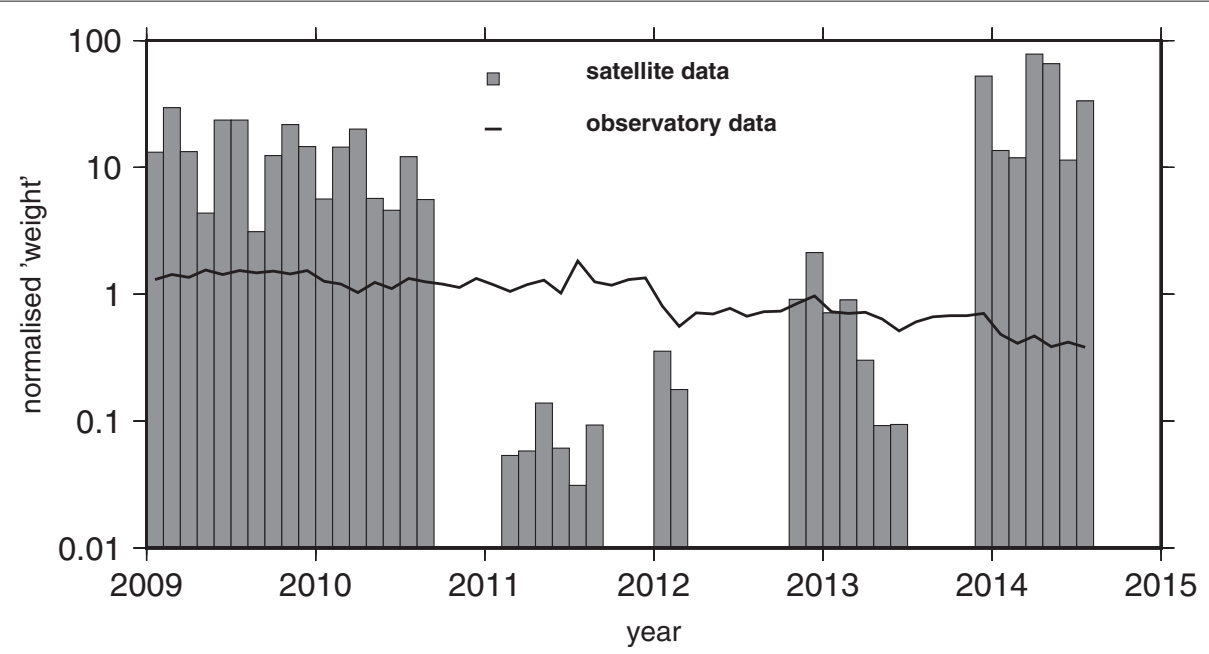

Figure 3 Approximate 'weight' of data in model. The 'weight' is computed from the sum of the inverse of the datum variances over 0.1 -year intervals from satellites (Ørsted, CHAMP, and Swarm) and observatories. The 'weight' is normalised to the mean of the observatory weights over all 0.1 -year intervals $(=1)$.

Future work will focus on estimating higher-degree external signals to improve their separation from internal sources.

This model described is fitted to the data by minimising $\Phi$ : the weighted sum of the squared model data residuals, the second time derivative of the radial field at the coremantle boundary (CMB), and the third time derivative of the radial field at the Earth's mean radius following Lesur et al. (2008):

$$
\Phi=\mathbf{e}^{T} \mathbf{C e}+\lambda_{1} \mathbf{m}^{T} \Lambda_{1} \mathbf{m}+\lambda_{2} \mathbf{m}^{T} \Lambda_{2} \mathbf{m}
$$

where $\mathbf{m}^{T} \Lambda_{1} \mathbf{m}=\left|\partial^{2} B_{r} / \partial t^{2}\right|_{r=\mathrm{CMB}}^{2}$ and $\mathbf{m}^{T} \Lambda_{2} \mathbf{m}=$ $\left|\partial^{3} B_{r} / \partial t^{3}\right|_{r=a}^{2}$. We note that variations on this scheme (e.g. Olsen et al. (2014)) and additional norms (e.g. maximum entropy in Finlay et al. (2012)) are also in use. The damping by radial derivative is necessary to control the behaviour of the order 6 B-splines, especially over the period 2011 to 2014 where global vector data are sparse. The relative weight of each term in the minimisation is controlled by two damping parameters associated with each of the derivatives of the radial field, $\lambda_{1}$ and $\lambda_{2}$. Testing (not shown) with different damping parameters has

Table 2 Mean and root mean square misfits between parent model estimate and input data

\begin{tabular}{|c|c|c|c|c|c|c|c|c|c|}
\hline \multirow[b]{2}{*}{ Source } & \multirow[b]{2}{*}{ Number of data } & \multicolumn{3}{|c|}{ Mean (nT) } & \multirow[b]{2}{*}{$F$} & \multicolumn{3}{|c|}{ Root mean square (nT) } & \multirow[b]{2}{*}{$F$} \\
\hline & & $X$ & $Y$ & $Z$ & & $X$ & $Y$ & $Z$ & \\
\hline CHAMP & $1,332,591$ & 0.10 & -0.19 & 0.05 & - & 25.20 & 27.64 & 12.25 & - \\
\hline Swarm A & 455,091 & -0.10 & -0.10 & 0.44 & - & 34.34 & 40.15 & 13.23 & - \\
\hline Swarm B & 426,324 & -0.45 & -0.09 & 0.17 & - & 35.06 & 40.55 & 12.73 & - \\
\hline Swarm C & 407,166 & -0.26 & -0.18 & -0.21 & - & 34.92 & 40.49 & 13.18 & - \\
\hline Observatories & 290,532 & 0.04 & -0.00 & 0.01 & - & 3.90 & 3.26 & 3.24 & - \\
\hline Ørsted & 281,967 & - & - & - & 0.75 & - & - & - & 7.65 \\
\hline CHAMP & 20,740 & - & - & - & -2.36 & - & - & - & 10.34 \\
\hline Swarm A & 749 & - & - & - & -4.42 & - & - & - & 14.41 \\
\hline Swarm B & 2,544 & - & - & - & -4.96 & - & - & - & 18.07 \\
\hline Swarm C & 1,196 & - & - & - & -2.91 & - & - & - & 14.64 \\
\hline Observatories & 63,830 & - & - & - & -0.07 & - & - & - & 18.89 \\
\hline
\end{tabular}

Satellite $F$ misfits are calculated with respect to scalar input data. Observatory $F$ misfits are at high geomagnetic dipole latitude and are calculated with respect to vector data projected onto an a priori model. All observatory vector misfits are at mid and low geomagnetic dipole latitudes. Mid and low latitudes are defined as being between $\pm 50^{\circ}$, with high latitudes outside this range. The number of data quoted counts each component separately. 
shown that a weighting of 0.4 for both these terms gives a robust fit to the data.

\section{Derivation of IGRF candidate models}

The DGRF candidate model can be generated at 2010.0 from the parent model's core field coefficients with Bspline representation up to degree 13 .

For the IGRF and 2015-2020 secular variation models, some extrapolation of the coefficients is necessary as the parent model does not extend beyond 2014.7. Furthermore, in order to reduce the influence of end effects, model snapshots are not extracted beyond 2014.5.

In global field modelling, extrapolation of the field is usually based upon some mathematical extrapolation of the time dependence of the coefficients. Whilst this is simple to implement, we have chosen instead to use a physics-based approach by modelling the flows in the Earth's liquid outer core and use these flows with certain assumptions to predict the field at 2015.0 and beyond. By assuming that the main field is essentially 'frozen' into liquid at the top of the outer core, it is possible to deduce the flow causing the observed field change at the surface of the Earth from an estimation of the relative changes over a period of a year or longer.

At large spatial scales, advection dominates diffusion on short time scales ( $<10$ years), though at sufficiently small spatial scales, diffusion does become important again. By neglecting diffusion, the radial part of the frozen-flux induction equation describing how flowing fluid advects the magnetic field can be written as (e.g. Holme (2007)):

$$
\dot{B}_{r}=-B_{r} \nabla_{H} \cdot \mathbf{u}-\mathbf{u} \cdot \nabla_{H} B_{r}
$$

where $\dot{B}_{r}$ is the time derivative of the radial magnetic field, $\nabla_{H}$ is the horizontal derivative operator, and $\mathbf{u}$ is the flow velocity containing the flow components in the north $(\theta)$ and east $(\phi)$ directions along the surface of the core mantle boundary (Whaler 1986).

Equation 7 implies that variation of the field is caused by the fluid pushing the magnetic field. We wish to solve for the velocity vector $\mathbf{u}$. In addition, we also wish to solve for the acceleration of the magnetic field in order to model the rate of change of the flow velocity. This can be described by the following equation:

$$
\ddot{B}_{r}=-\dot{B}_{r} \nabla_{H} \cdot \mathbf{u}-B_{r} \nabla_{H} \cdot \dot{\mathbf{u}}-\dot{\mathbf{u}} \cdot \nabla_{H} B_{r}-\mathbf{u} \cdot \nabla_{H} \dot{B}_{r}
$$

where $\ddot{B}_{r}$ is the second time derivative of the radial magnetic field and $\dot{\mathbf{u}}$ is the flow acceleration.

Together the flow velocity and acceleration can describe the observed spatial change of the main field over time. At large horizontal spatial scales $(>1,000 \mathrm{~km})$, an average flow velocity of around $20 \mathrm{~km} /$ year is observed while the flow acceleration has a mean of about $2 \mathrm{~km} / \mathrm{year}^{2}$.
As the secular variation and acceleration of the magnetic field can be measured at the surface, it remains then to solve for the flow velocity and acceleration using the mathematical relationship between fluid flow and magnetic field, and applying inverse theory. However, in detail, the solution to Equations 7 and 8 for $\mathbf{u}$ and $\dot{\mathbf{u}}$ are ambiguous as there are two unknowns in each equation (i.e. the velocity/acceleration in the north and east directions). To reduce the ambiguity, additional constraints are required. We apply a constraint that assumes the flow velocity remains constant (steady) over a short period of time. It can be shown that the solution of a steady flow is unique, as long as at least three time steps are used (Waddington et al. 1995). In a similar manner to constant flow, the steady acceleration of the flow can be deduced by combining the acceleration over that number of years. The steady flow captures the gross large-scale aspects of magnetic field change but does not allow any rapid short-term features. Adding in a component of acceleration allows a better description of the (slightly) non-linear change of the magnetic field over the short period. We note the detailed implementation of the technique and evidence for the usefulness of core flows in secular variation forecasting is given in Whaler and Beggan (2015).

For our core flow model, the Gauss coefficients of the parent of the IGRF-12 candidate field model from 2009.0 to 2014.5 were used to compute the secular variation and secular acceleration of the field, initially. The flow velocity and acceleration coefficients are related to the magnetic field via a set of equations which involve integrals of triple products of spherical harmonics and their spatial derivatives and the main field coefficient (Whaler 1986). The solutions require three damping parameters to impose a spatial smoothness on the resulting steady flow velocity and acceleration solutions. These parameters were picked by tuning the output using retrospective data fitting to prior magnetic field data from an a priori model and observatory data. We assume the SV 'observation errors' on the Gauss coefficients to be on the order of $1 \mathrm{nT} /$ year in the inversion. However, we use an L1-norm iterative reweighting scheme to reduce the effect of any large outliers when fitting the model back to the data. We also truncate the flow model at degree 14 and damp using the Bloxham 'strong' norm (Bloxham 1988) which effectively reduces the influence of the model SV above degree 8 . We ignore the 'subgrid' processes which we assume to be small for the purposes of this flow modelling (Pais and Jault 2008).

The forecasting process starts with the last epoch of the main field derived from satellite and observatory data (i.e. 2014.5). For this time point, the secular variation and secular acceleration of the field are computed from the steady flow velocity and acceleration models. Equations 7 and 8 show that there is a dependence of the secular 

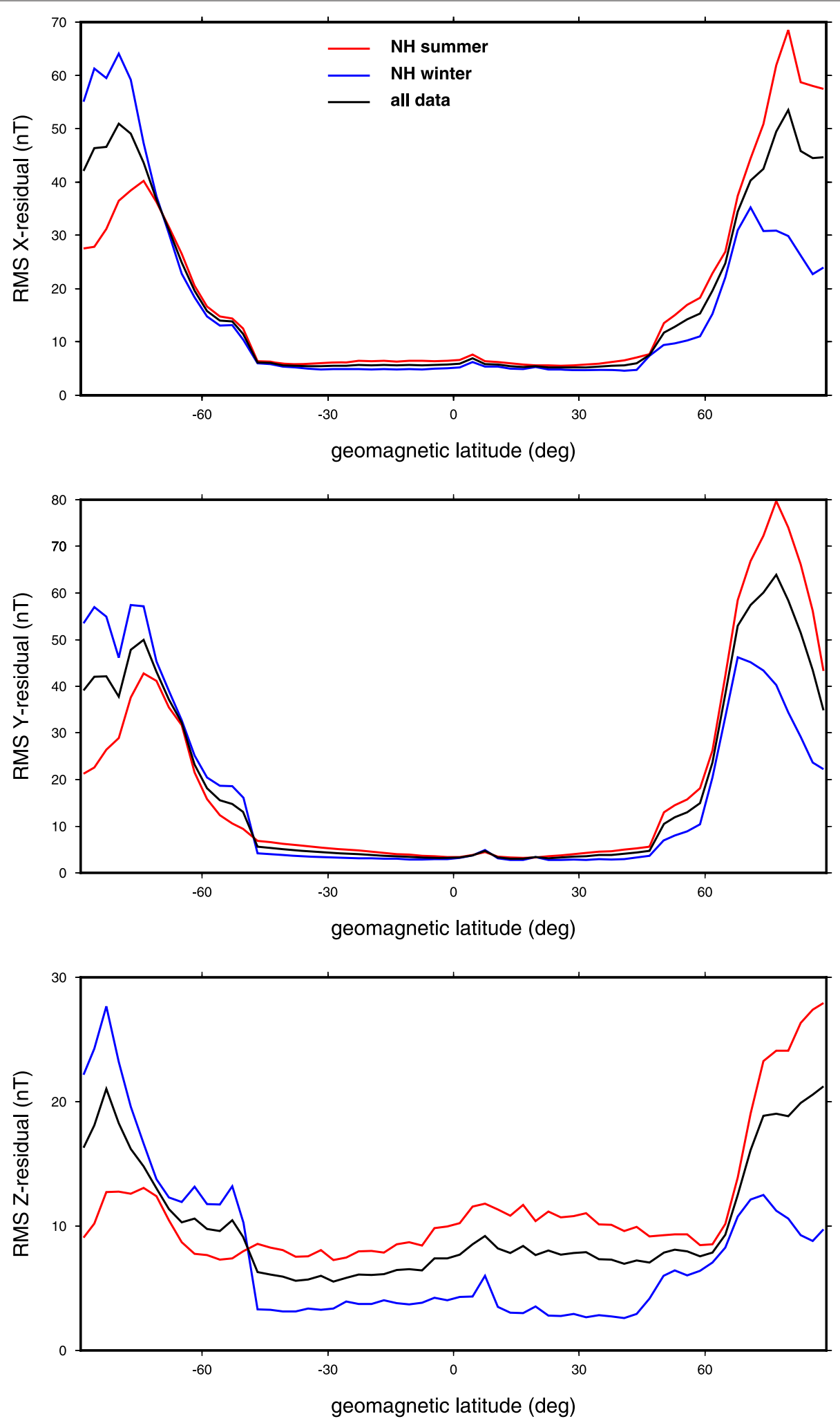

Figure 4 Root mean square of residuals between all input satellite vector data and parent model. Lines show $X, Y$, and $Z$ component residuals plotted against geomagnetic dipole latitude. Northern hemisphere summer is defined as spring through autumn equinox, northern hemisphere winter as autumn through spring-equinox. The RMS values are calculated over $3^{\circ}$ wide latitude bands. Note that the drop in residuals between $\pm 50^{\circ}$ is due to local time selection at those latitudes. 

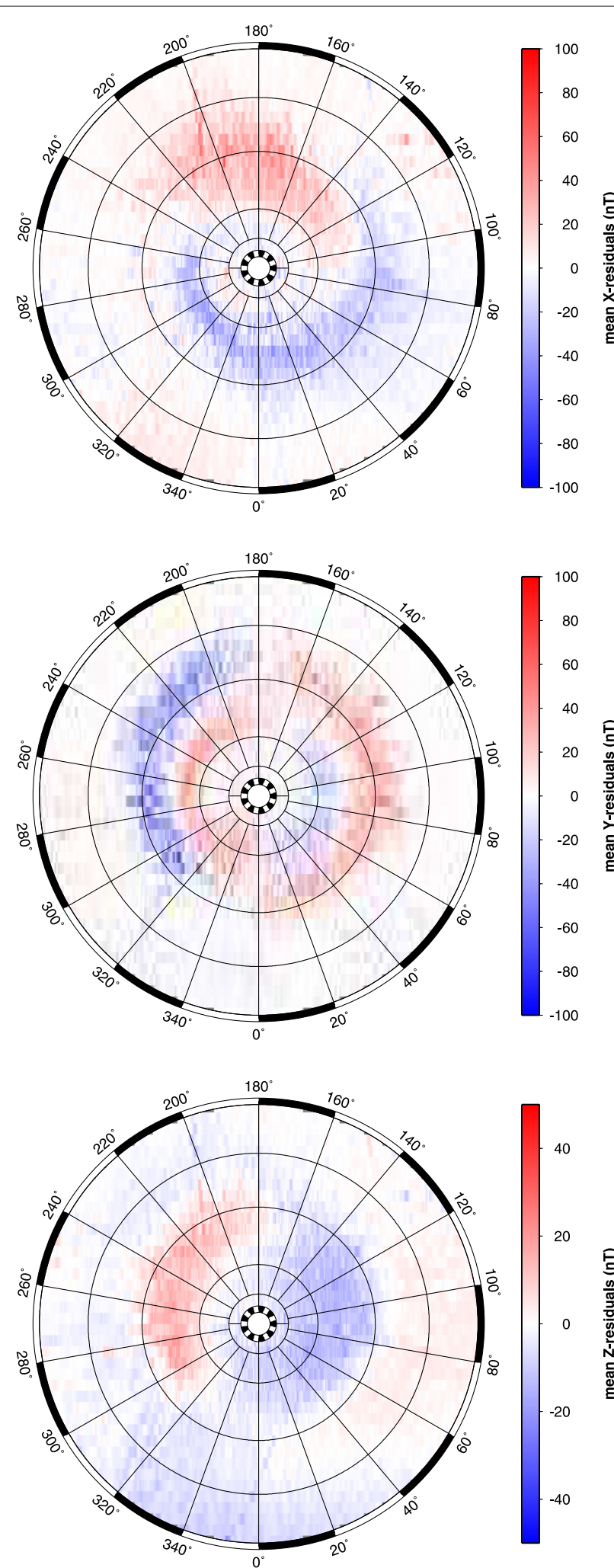

40

20

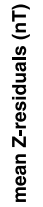

Figure 5 Mean of the residuals between parent model and input satellite data for each magnetic component. Top: $X$, middle: $Y$, and bottom: $Z$ over northern polar cap $\left(\geq 50^{\circ}\right.$ geomagnetic dipole latitude) over the entire input data set. The plot is ordered by Solar Magnetic longitude and latitude with the Sun in the direction of zero longitude and $90^{\circ}$ latitude aligned with the north geomagnetic dipole pole. Patterns of sun-synchronous current systems are clearly visible. 
variation and secular acceleration on the main field itself. Hence, the process is non-linear and has to be stepped forwards at a suitably fine time resolution, in this case, 1 month (Beggan and Whaler 2010). Each month, a new set of matrices relating flow velocity and acceleration to secular variation and secular acceleration are computed based on the main field and secular variation coefficients. This generates a set of predicted secular variation and secular acceleration coefficients, which are added back to the main field coefficients to give the prediction of the main field for the following month. The Gauss coefficients of the main field were advected forward from 2014.5 to 2020.0. The coefficients of the average annual secular variation (in $\mathrm{nT} /$ year) were calculated from the difference of the main field models at 2020.0 and 2015.0, divided by 5 years.

\section{Results and discussion}

\section{Parent model}

The (unweighted) mean and root mean square (RMS) of the misfits are summarised in Table 2. Direct comparison between CHAMP, Ørsted, Swarm, and observatory misfits is not straightforward. The date range covered by the sources vary, and some do not overlap in time (see Figure 1). Only observatory data are available through the period covered by the parent model. Swarm data do not

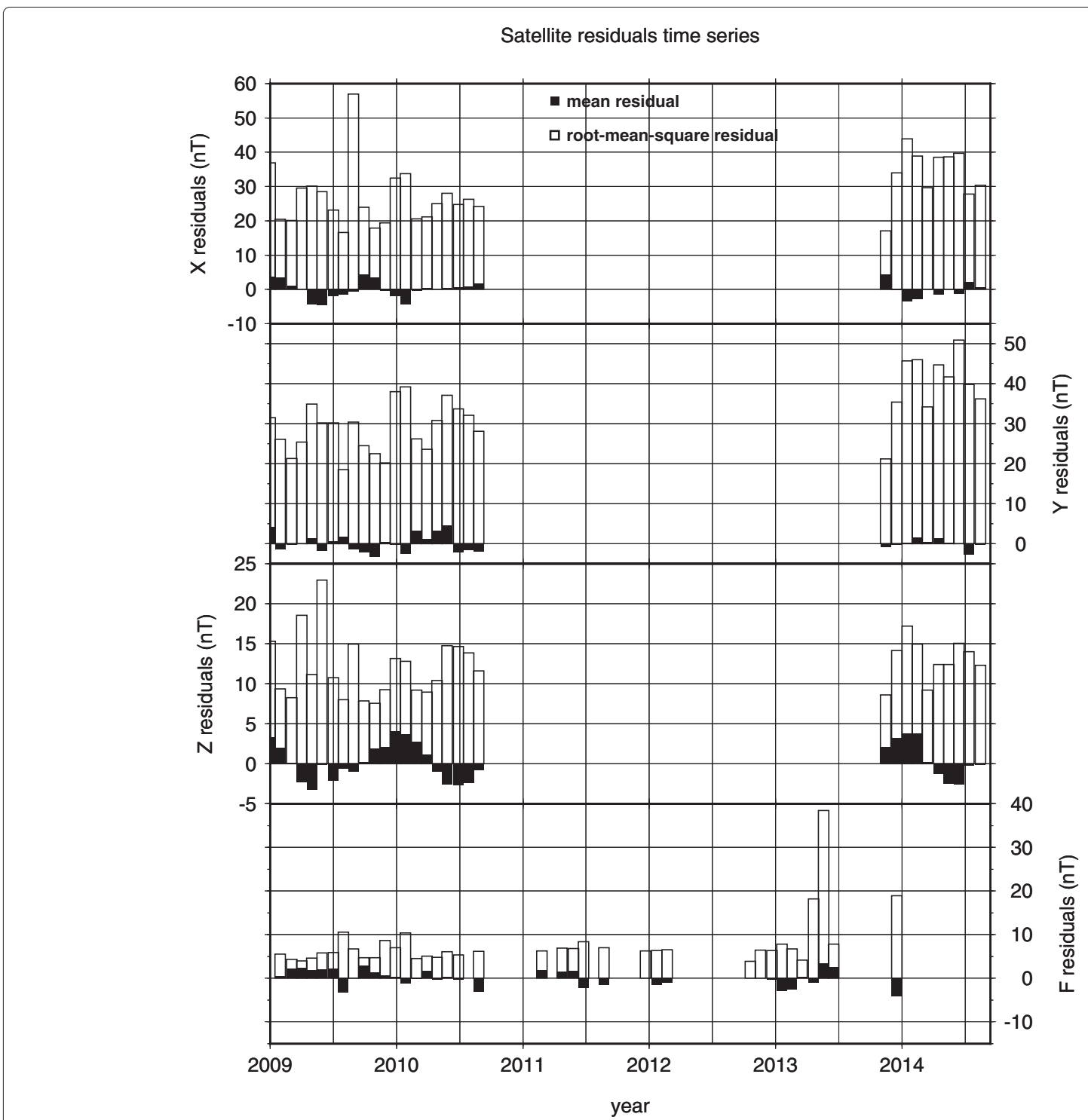

Figure 6 Mean and root mean square of the residuals between parent model and input satellite data. Values for each magnetic component are calculated within within successive 30-day periods. To preserve statistical robustness, periods with fewer than $10 \%$ of mean number of data per period (approximately 3,000 for $X, Y$, and $Z$, and approximately 600 for $F$ ) are not plotted. 
overlap at all with Ørsted and CHAMP data, and Ørsted overlaps with CHAMP data only at the start of the model. Even then, the types of data are different: over the period covered by the parent model, Ørsted is only providing scalar data. And while vector and/or scalar satellite data are selected at all latitudes, observatory true-vector (as opposed to pseudo-scalar) data are only used at mid and low latitudes.

However, it is worth commenting on some patterns in the misfits. The mean misfits are all very small. For the observatory data, crustal bias parameters are effectively free (static) parameters for each observatory, which will act to remove any mean residuals. However, non-zero mean misfits are still possible here, and especially with the satellite data, due to unmodelled sources and the damping scheme used to produce the model.

The RMS residuals are lowest in $X, Y$, and $Z$ for the observatory data, but this is due to those data being at mid and low latitudes, where the data will be less contaminated by unmodelled sources of the field, especially auroral current systems. The RMS $F$ residuals for the observatory (pseudo-) scalar data, which are all at high latitudes, are larger than those from satellite scalar data from all latitudes. The dominant contribution of high-latitude residuals to the RMS misfits can be seen in Figure 4 for all components, although it is reduced in winter time. A map of these misfits in the northern polar regions, ordered by (sun-synchronous) solar magnetic longitude and latitude is shown in Figure 5 for each component. This clearly shows the pattern of auroral currents. The extremes of the mean $X$ and $Y$ misfits in Figure 5 and the corresponding RMS misfits in Figure 4 all show the dominance of those components over the $Z$ component at high latitudes due to field-aligned currents, and this is reflected in the global RMS misfits in Table 2.

The mean and RMS residuals versus time for each component $(X, Y, Z$, and $F)$ are shown in Figure 6 for satellite data. The gap in satellite data, and vector data in particular, through 2011 to 2014 make patterns difficult to spot. However, the RMS are slightly larger towards the end of the time series in the Swarm era compared with the earlier CHAMP and Ørsted data. This is likely due to the increase in geomagnetic activity in the later data around solar maximum (approximately 2014). The mean residuals also show some periodic variation, particularly in the $Z$-component, which appear roughly annual although continuous data extend less than 2 years and the local time evolution of satellite orbits could also play a role. However, supporting evidence for an annual signal is shown in Figure 7, which plots the mean $Z$-residual versus geomagnetic latitude for summer and winter seasons. The mean residuals are clearly largest over the respective summers of the northern and southern auroral regions. These are predominantly negative in northern hemisphere summer and positive in southern hemisphere summer, and much smaller in winter, which is consistent with Figure 6. Our decision to include sunlit auroral region data has significantly increased the noise level of the data in those areas, but it is important to note that data in the auroral regions are down-weighted by the LAVA index and along-track standard deviation for precisely this reason. Comparisons with similar versions of our parent model produced without these data (not shown) support our decision to avoid large seasonal data gaps.

The mean and RMS misfits for the observatory data are shown in Figure 8. The RMS misfits also show a slight

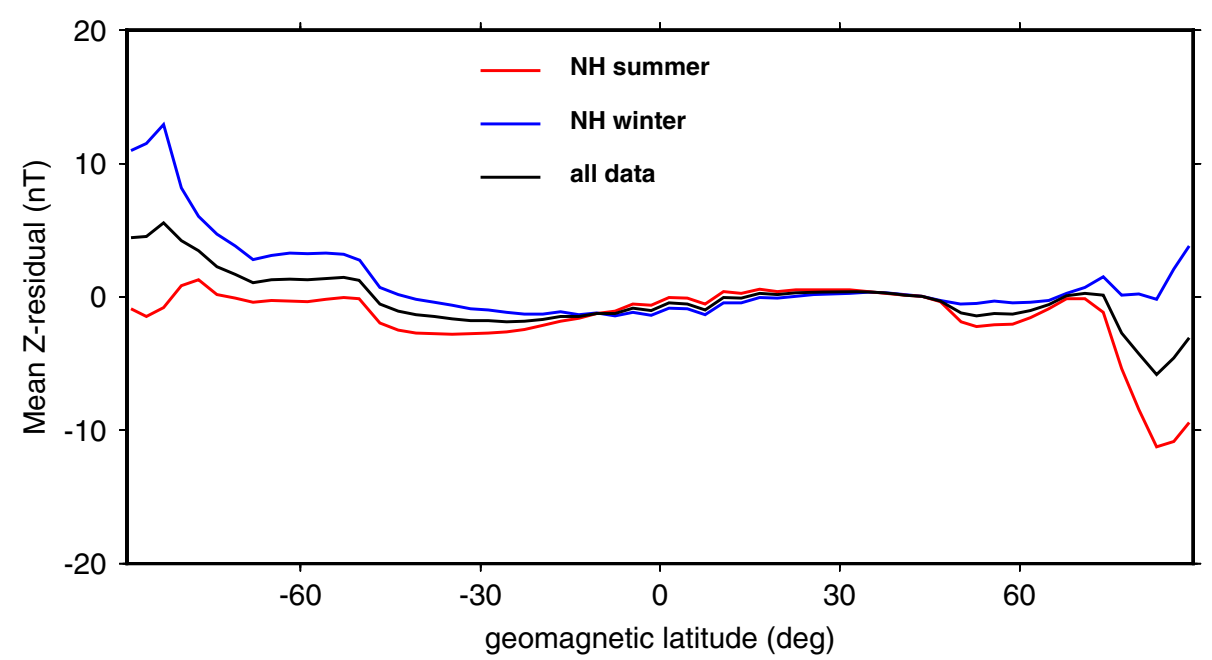

Figure 7 Mean residuals between Z-component of vector satellite data and parent model estimate. Values are plotted versus geomagnetic dipole latitude. Northern hemisphere summer is defined as spring through autumn equinox, northern hemisphere winter as autumn through spring equinox. The mean values are calculated over $3^{\circ}$ wide latitude bands. 
Observatory residuals time series

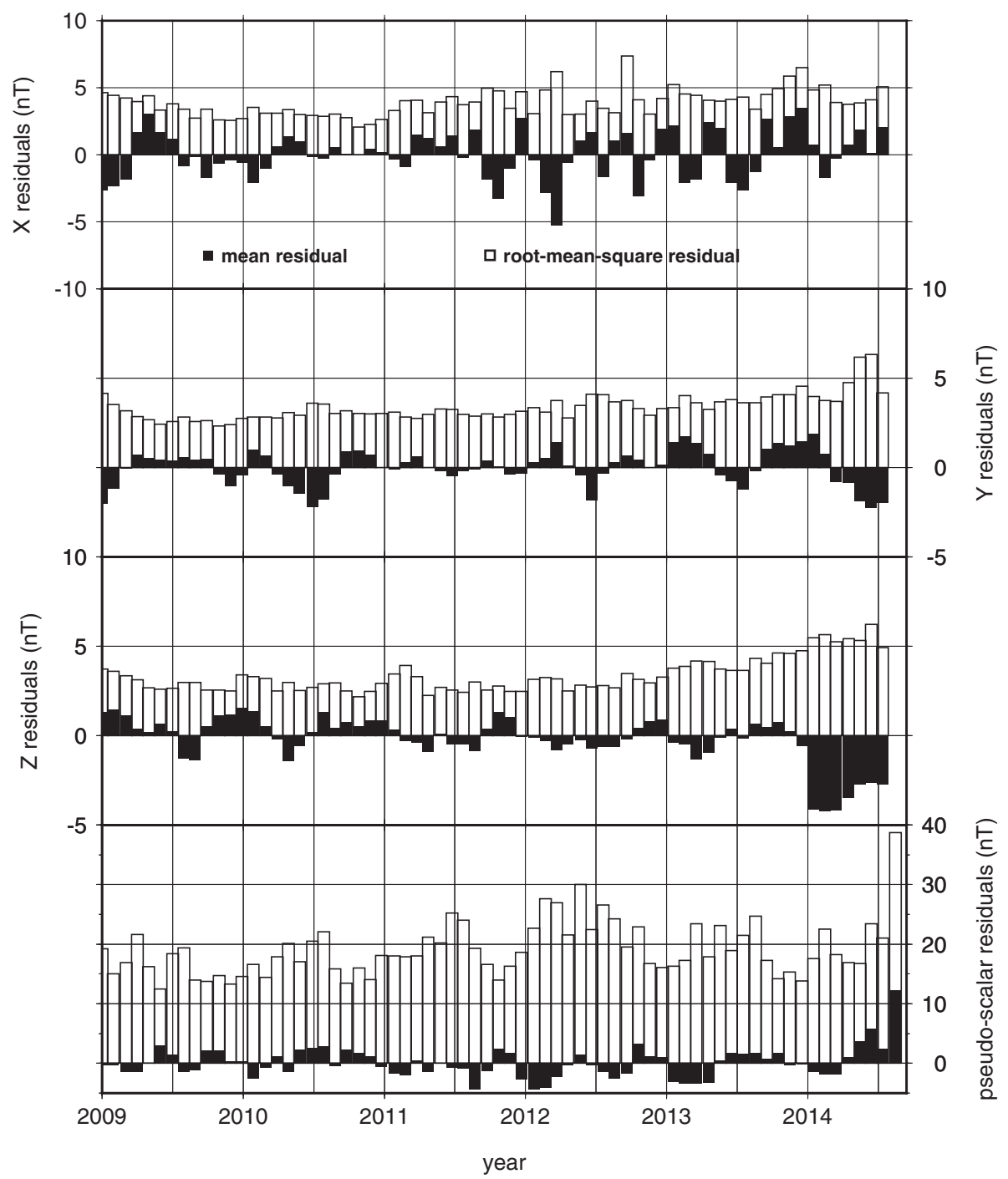

Figure 8 Mean and root mean square of the residuals between parent model and input observatory data. Values for each magnetic component are calculated within successive 30-day periods. Periods with fewer than $10 \%$ of mean number of data per period (approximatey 150 for $X, Y$, and $Z$, and approximatey 100 for $F$ ) are not plotted to preserve statistical robustness. Vector data are all within $\pm 50^{\circ}$ geomagnetic dipole latitude and all (pseudo-) scalar data are outside this range.

increase in size over the latter half of the time series, but the difference is not large over most of the data set. Towards the very end of the time series, the magnitude of the mean misfits do increase noticeably for the $Z$ component, which coincides with the start of 2014. The reason for this may be related to a switch from definitive to quasi-definitive data (e.g. Clarke et al. (2013)), which, given the small residual mean, requires only a slight drop in data quality from a small number of observatories. There is also some evidence of periodic, possibly annual, signals in these data, too.

The main field, secular variation, and secular acceleration spectra, extracted at yearly intervals from the parent model, are shown in Figure 9. The main field spectra exhibit a strict monotonic decline with increasing degree for all snapshots. Changes between the spectra over the 5year span are relatively small (top panel). The secular variation, extracted from the spline model at the same dates, shows a fairly steady decline in power with increasing degree (middle panel). The greatest year-to-year variation is in the lower degrees with very little above degree 6 . This is in marked contrast to the behaviour seen in the parent model for BGS's IGRF-11 candidates (Hamilton et al. 2010), in which the variability increased with degree. The reason for this is the switch to a spline time dependence with explicit damping of higher-order rates of change 

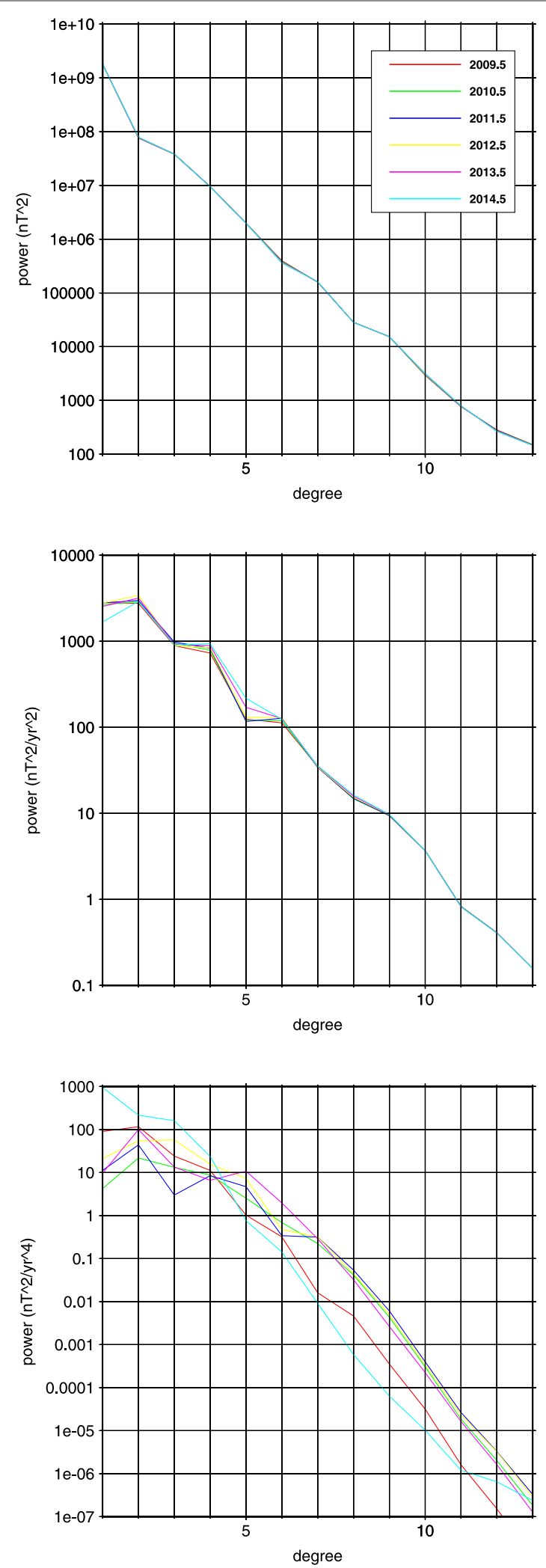

Figure 9 Spectral power per degree extracted from parent model at yearly intervals from 2009.5 to 2014.5. The plots show the spectra for the main field (top), secular variation (middle), and secular acceleration (bottom). The effect of temporal damping is visible in the smooth, monotonically decreasing behaviour of the higher-degree secular variation and secular acceleration power. 
$\left(\partial^{2} / \partial t^{2}\right.$ and $\left.\partial^{3} / \partial t^{3}\right)$. This is confirmed by the rapid drop off of secular acceleration power with increasing degree in Figure 9 (bottom panel). As was argued in Hamilton et al. (2010), the undamped secular acceleration above about degree 8 was dominated by noise, and so the damping used seems justified.

\section{Comparison with other candidates}

Table 3 shows the global RMS differences between the BGS candidate, derived from the parent model and coreflow prediction, and those of other institutes, computed at the reference radius of $6,371.2 \mathrm{~km}$. As expected, the DGRF models agree better than the IGRF or SV. The BGS candidate DGRF matches the DTU, NGDC, and GFZ models very well, with less than 3-nT RMS difference. Figure 10 shows the RMS differences per degree, and again, the BGS model agrees well with the DTU, NGDC, and GFZ models and is clearly not an outlier amongst the other candidates.

The differences between IGRF models in Table 3 and Figure 10 are larger than for the DGRF, but the good agreement with DTU, NGDC, and GFZ models remains; there is also reasonably good agreement with the IPGP model. Although the BGS model differs with most models around degree 5 specifically, the overall RMS differences are generally low.

The largest differences between the models are for the 2015-2020 mean secular variation estimates. The different institutes use a range of techniques to predict the secular variation including physical versus mathematical extrapolations. Figure 10 again shows that although there are differences with other models, especially at degree 5 , the BGS model is well within the range of variability of other models.

Table 3 Global root mean square differences

\begin{tabular}{lccc}
\hline \multirow{2}{*}{ Model } & \multicolumn{3}{c}{$\begin{array}{c}\text { Root mean square difference } \\
\text { with BGS candidates }\end{array}$} \\
\cline { 2 - 4 } & $\begin{array}{c}\text { DGRF } \\
\mathbf{2 0 1 0} \text { (nT) }\end{array}$ & $\begin{array}{c}\text { IGRF } \\
\mathbf{2 0 1 5} \text { (nT) }\end{array}$ & $\begin{array}{c}\text { SV 2015-2020 } \\
\text { (nT/year) }\end{array}$ \\
\hline DTU Space & 2.8 & 6.8 & 9.7 \\
ISTerre & 6.6 & 12.1 & 14.2 \\
IZMIRAN & 4.0 & 14.1 & 16.6 \\
NGDC Boulder & 2.4 & 7.3 & 11.0 \\
GFZ Potsdam & 2.6 & 6.3 & 10.9 \\
NASA/GSFC & 4.9 & - & 11.6 \\
IPGP/CEA-LETI/CNES & - & 9.1 & 10.7 \\
LPG Nantes & - & 10.3 & 14.1 \\
ETH Zurich & - & 16.2 & - \\
\hline
\end{tabular}

Differences at reference radius $(6,371.2 \mathrm{~km})$ between BGS candidate models and candidates from other contributing institutes.
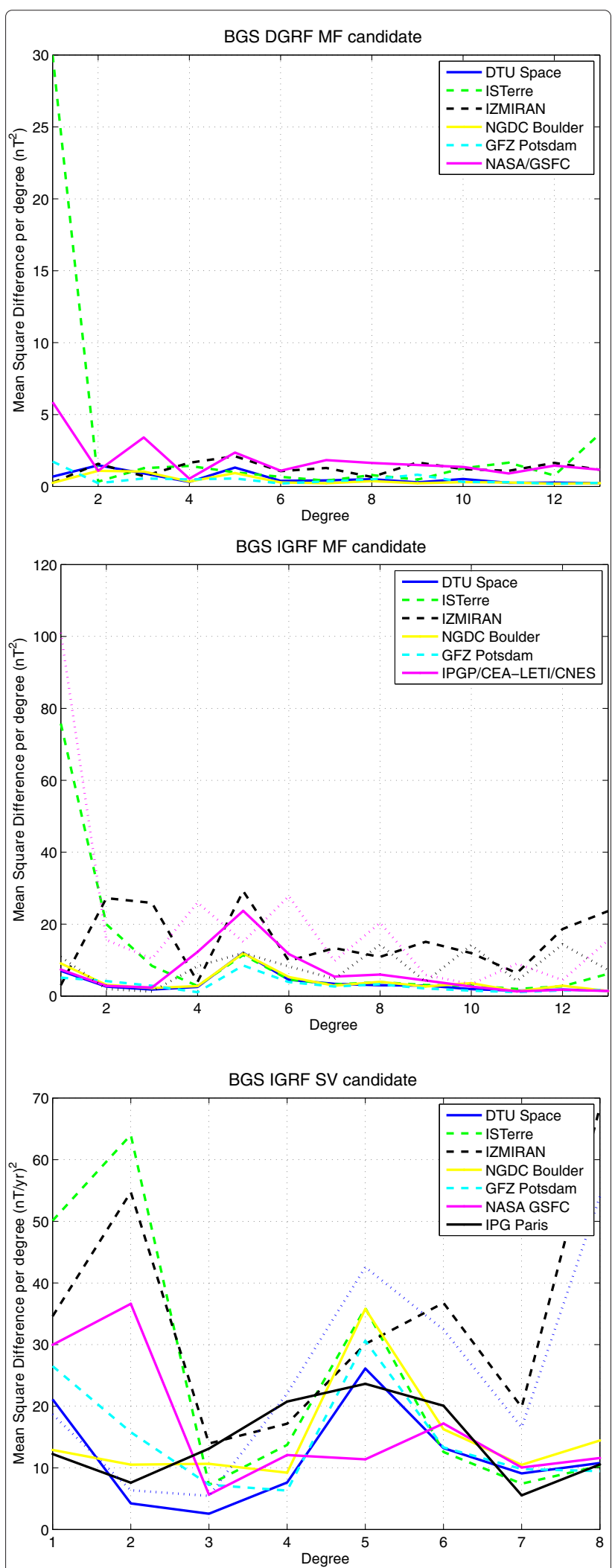

Figure 10 Mean squared difference per degree between BGS candidate model and those from other institutions. Top: DGRF2010, middle: IGRF 2015 and bottom: mean secular variation 2015-2020. 


\section{Conclusions}

The BGS parent model has successfully used the most up-to-date data available from Swarm, observatory, and Ørsted as well as those from the last phase of the CHAMP mission. Inclusion of all these data sources, especially the observatory data, combined with a regularised model of time dependence using B-splines have allowed BGS to produce a robust model of the core field over the past 6 years. These data have also allowed us to estimate the core flow over this period and to provide a physically based prediction of the secular variation over the lifetime of IGRF-12. Comparisons have shown the BGS candidates to be consistent with those of other contributing institutes.

\section{Competing interests}

The authors declare that they have no competing interests.

\section{Authors' contributions}

$\mathrm{BH}$ carried out the satellite data selection. VAR developed and produced the parent model. CDB produced the core-flow model and secular variation prediction. SM carried out the observatory data selection and quality control. All the authors also contributed to all parts of the paper. All authors read and approved the manuscript.

\section{Acknowledgements}

We would like to acknowledge the Ørsted, CHAMP, and Swarm science data centres, INTERMAGNET (at http://www.intermagnet.org), our colleagues in BGS who run the World Data Centre for Geomagnetism, and the many individual institutes operating observatories. We would also like to thank the referees for their comments and suggestions that have improved the paper. This paper is published with the permission of the Executive Director of the British Geological Survey (NERC).

Received: 29 January 2015 Accepted: 10 April 2015

Published online: 12 May 2015

\section{References}

Beggan C, Whaler K (2010) Forecasting secular variation using core flows. Earth Planets Space 62(10):821-828. doi:10.5047/eps.2010.07.004

Beggan CD, Macmillan S, Hamilton B, Thomson AWP (2013) Independent validation of Swarm Level 2 magnetic field products and 'Quick Look' for Level $1 \mathrm{~b}$ data. Earth Planets Space 65:1345-1353. doi:10.5047/eps.2013.08.004

Bloxham J (1988) Chapter 9. The determination of fluid flow at the core surface from geomagnetic observations. In: Vlaar NJ, Nolet G, Wortel MJR, Cloetingh SAPL (eds). Mathematical geophysics. D. Reidel Publishing Company, Dordrecht. pp 189-208

Clarke E, Baillie O, Reay SJ, Turbitt CW (2013) A method for the near real-time production of quasi-definitive magnetic observatory data. Earth Planets Space 65(11):1363-1374. doi:10.5047/eps.2013.10.001

Finlay CC, Maus S, Beggan CD, Bondar TN, Chambodut A, Chernova TA, Chulliat A, Golovkov VP, Hamilton B, Hamoudi M, Holme R, Hulot G, Kuang W, Langlais B, Lesur V, Lowes FJ, Lühr H, MacMillan S, Mandea M, McLean S, Manoj C, Menvielle M, Michaelis I, Olsen N, Rauberg J, Rother M, Sabaka TJ, Tangborn A, Tøffner-Clausen L, Thébault E, et al. (2010) International Geomagnetic Reference Field: the eleventh generation. Geophys J Int 183:1216-1230. doi:10.1111/j.1365-246X.2010.04804.x

Finlay CC, Jackson A, Gillet N, Olsen N (2012) Core surface magnetic field evolution 2000-2010. Geophys J Int 189:761-781. doi:10.1111/j.1365-246X.2012.05395.x

Hamilton B, MacMillan S, Thomson A (2010) The BGS magnetic field candidate models for the 11th generation IGRF. Earth Planets Space 62:737-743. doi:10.5047/eps.2010.05.005

Holme R (2007) Large scale flow in the core. In: Olson P, Schubert G (eds). Treatise on Geophysics. Elsevier, Amsterdam Vol. 8. pp 107-130

Lesur V, Macmillan S, Thomson AWP (2005) The BGS magnetic field candidate models for the 10th generation IGRF. Earth Planets Space 57:1157-1163
Lesur V, Wardinski I, Rother M, Mandea M (2008) GRIMM: the GFZ Reference Internal Magnetic Model based on vector satellite and observatory data. Geophys J Int 173:382-394

Lesur V, Wardinski I, Hamoudi M, Rother M (2010) The second generation of the GFZ Reference Internal Magnetic Model: GRIMM-2. Earth Planets Space 62:765-773. doi:10.5047/eps.2010.07.007

Lühr H, Maus S (2010) Solar cycle dependence of quiet-time magnetospheric currents and a model of their near-Earth magnetic fields. Earth Planets Space 62:843-848. doi:10.5047/eps.2010.07.012

Macmillan S, Olsen N (2013) Observatory data and the Swarm mission. Earth Planets Space 65:1355-1362. doi:10.5047/eps.2013.07.011

Maus S, Manoj C, Rauberg J, Michaelis I, Lühr H (2010) NOAA/NGDC candidate models for the 11th generation International Geomagnetic Reference Field and the concurrent release of the 6th generation Pomme magnetic model. Earth Planets Space 62:729-735. doi:10.5047/eps.2010.07.006

Olsen N, Lühr H, Finlay CC, Sabaka TJ, Michaelis I, Rauberg J, Tøffner-Clausen L (2014) The CHAOS-4 geomagnetic field model. Geophys J Int 197:815-827. doi:10.1093/gji/ggu033

Pais MA, Jault D (2008) Quasi-geostrophic flows responsible for the secular variation of the Earth's magnetic field. Geophys J Int 173:421-443. doi:10.1111/j.1365-246X.2008.03741.x

Sabaka TJ, Olsen N, Langel R (2002) A comprehensive model of the quiet-time, near-Earth magnetic field: phase 3. Geophys J Int 151:32-68

Thomson AWPT, Lesur V (2007) An improved geomagnetic data selection algorithm for global geomagnetic field modelling. Geophys J Int 169:951-963

Thomson AWP, Hamilton B, Macmillan S, Reay S (2010) A novel weighting method for satellite magnetic data and a new global magnetic field model. Geophys J Int 181:250-260

Waddington R, Gubbins D, Barber N (1995) Geomagnetic field analysis - V, Determining steady core-surface flows directly from geomagnetic observations. Geophys J Int 122(1):326-350. doi:10.1111/j.1365-246X.1995.tb03556.x

Whaler KA (1986) Geomagnetic evidence for fluid upwelling at the core-mantle boundary. Geophys J R Astr Soc 86:563-588

Whaler KA, Beggan CD (2015) Derivaton and use of core surface flows for forecasting secular variation. J Geophys Res 120:1400-1414. doi:10.1002/2014JB011697

\section{Submit your manuscript to a SpringerOpen ${ }^{\mathcal{O}}$ journal and benefit from:}

- Convenient online submission

- Rigorous peer review

- Immediate publication on acceptance

- Open access: articles freely available online

- High visibility within the field

- Retaining the copyright to your article

Submit your next manuscript at $>$ springeropen.com 